\title{
FHIR Tools for Healthcare Interoperability
}

\author{
Amit Walinjkar* and John Woods \\ Faculty of Computer Science and Electronics Engineering, University of Essex, UK
}

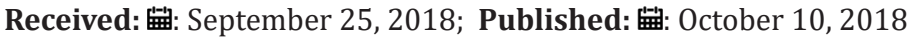

*Corresponding author: Amit Walinjkar, Faculty of Computer Science and Electronics Engineering, University of Essex, Colchester, Essex, UK

\begin{abstract}
Electronic Health Records (EHR) is an essential element in human healthcare monitoring systems these days. As a large amount of data continues being archived and uploaded to healthcare repositories, virtually every second across the globe, vast amount of data mining tasks continue being modelled and modified to extract valuable decision support information. The Health Level 7 (HL7) consortium provides the framework and related standards for the exchange, integration, sharing, and retrieval of electronic health information that supports clinical practice, management and delivery. With the large number of Internet of Things (IoT) health care kits becoming available it has become increasingly difficult to log the realtime patient monitoring information to healthcare repositories. As patients continue being monitored in real-time it has become essential that the trauma events information such as stroke or cardiac arrhythmia be uploaded to the EHR in real-time. Currently available monitoring devices can monitor and analyse an abnormal condition but may not be able to upload these events in real-time. The proposed research focused on developing real-time interoperability tools and services, which can enable wearable IoT devices to interact with the EHR in real-time and can provide real-time decision support.

The Fast Healthcare Interoperability Resources (FHIR) specification was used to develop and encode trauma related information in terms of FHIR resources, conceptual and logical models using clinFHIR tools. A HAPIFHIR application was implemented on an IoT device which could upload real-time ECG, PPG and relevant trauma information on a test FHIR server. The HAPIFHIR application code could encapsulate ECG arrhythmia, vital signs and trauma events in a single observation and could upload it to the HAPIFHIR server. Several such observations could be linked to a patient context and could be observed in real time in EHR. The ECG, the PPG, vital signs and trauma events were encoded according to Systematized Nomenclature of Medicine - Clinical Terms (SNOMED-CT) specifications. The alerts and alarms mechanism could assist the emergency response teams at the hospitals to prepare for an emergency well in time. An analogue front-end biomedical device was used for data acquisition and signal processing and the IoT devices were networked over wireless network to upload the events and observations to the FHIR server in real time. The system focussed on 'preventive care' as the next generation personalized health-care monitoring devices continue becoming available.
\end{abstract}

Keywords: IoT Healthcare; Trauma analysis; HL7; ECG FHIR; SNOMED-CT FHIR; HAPI FHIR; clinFHIR

\section{Introduction}

There are commercially available health monitoring kits for example the Holter ECG kit which are capable of monitoring a patient patient's heart rhythms for 24 to 48 hours during normal human activity [1]. The kit however only records the ECG and it does not analyse the heart rhythm to detect heart arrhythmia. The ECG recordings have to be manually analysed by an expert cardiologist. There are several such kits like the Alivecor and the Shimmer Sensing kits [2,3] which effectively monitor heart conditions though do not upload the traumatic events related information that a patient may have undergone. The system proposed in this research consists of a software implemented on the wearable IoT device which encapsulates the sample ECG readings in a standard XML or JSON format [4] and transmits the samples to the analytical server that uploads the FHIR payload to the FHIR server after performing the trauma analysis. Attempts have been made on previous occasions to make real time updates to electronic health records using internet client server technologies [5], though to develop such an infrastructure is however, a government policy decision rather than an implementation exercise.

HL7 (Health Level-7) is a standards agency that develops standards for electronic health resources and FHIR is one such standard that enables Electronic Health Records (EHR) interoperability through web-service interfaces is [6] HL7 FHIR Web services. An overview of SNOMED-CT coding schemes along with interrelationships between clinical codes has been provided in this paper. An Oracle Java based FHIR test server and HAPIFHIR client based implementation has also been explained. In the methods section a detailed implementation of a HAPIFHIR client has been explained. The usage of clinFHIR tool which models codable concepts, resources, and elements, components using scenario modeller, logical modeller and conceptual modelling tools has been demonstrated. SNOMED CT is a coding system that 
describes clinical concepts, vocabulary, descriptions and interrelationships between clinical codes. The results and discussions section discusses the advantages of using the FHIR based client application and FHIR modelling tools to develop an IoT-based realtime decision health support system.

\section{Overview of FHIR Healthcare Interoperability Workflow}

\section{Signal Processing, Dataset Preparation and Analysis}

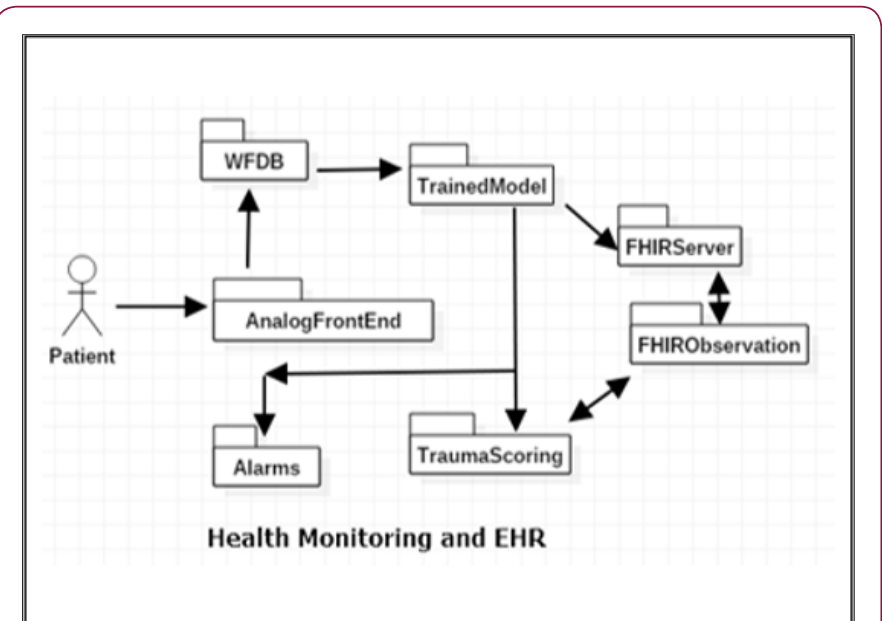

Figure 1: Health Monitoring in a typical IoT based wearable kit demonstrating data-acquisition to FHIR observation upload to FHIR Server.

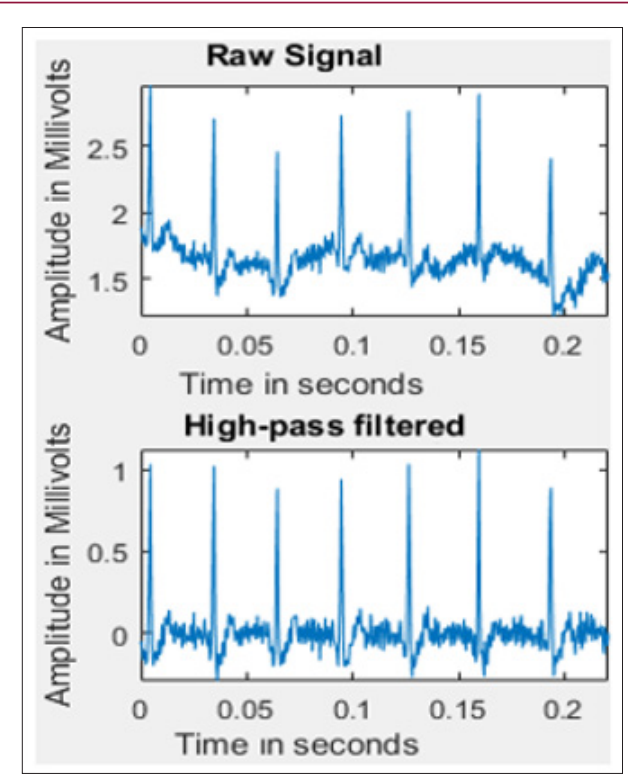

Figure 2: A noisy ECG waveform filtered using digital signal processing algorithms like Chebyshev II order Filter.

A complete workflow of the process from data acquisition to signal conditioning, followed by data analysis and abnormal event classification, further followed by upload of the real time health monitoring information to FHIR server is illustrated in Figure 1. A typical signal processing application involves data acquisition in the form of ECG signal samples being acquired from a human subject. These signals could be acquired from the human subject in noisy conditions where there is bioelectric signal interference and noise due to other electrical components. To remove the noise, signals are filtered using Common filtering techniques using digital filters like Butterworth and Chebyshev filters. There is also noise induced due to motion artefacts, e.g. due to the motion of the patient being monitored and muscular bioelectric signal interference. The filtered waveform extracted from the noisy signal after filtration process is shown in Figure 2. After the signal has been filtered, samples can be acquired to extract features of the waveform for further data analysis. The signals have to be acquired according to Nyquist criteria, where the sampling frequency should be at least half the bandwidth of the signal. The samples prepared are passed to data analysis stage. The data analysis stage typically involves training a machine learning model based on existing waveform data sets.

\section{HL7 FHIR Interoperability}

Fast Healthcare Interoperability Resources (FHIR) defines a set of "Resources" that represent clinical concepts. The resources can be coded and managed in isolation or aggregated into complex structures and documents which could be archived and queried as and when required. FHIR has been designed for the web; the resources are based on simple XML or JSON structures, with an http-based RESTful protocol and each resource is encapsulated and identified by a Uniform Resource Locator (URL).

\section{Methods to Implement FHIR on IoT Devices}

The implementation of interoperable component on IoT devices requires modelling FHIR resources, codes and if required codable concepts based on FHIR specifications. In a typical healthcare monitoring kit an analogue front-end device such as the 3-lead ECG sensor (Analog Devices AD8233) [7] is used for data acquisition. The data acquisition system is interfaced with their intelligent processor like the Texas Instruments (TI) Cortex A7 processor (TI Beaglebone Black, Texas Instruments, Digi-key, USA). The readings obtained from an analogue front-end was denoised filtered and conditioned to obtain a smooth waveform. The signal was converted from analogue to digital form and was passed to the intelligent processor like the Beaglebone black. Since the signals and the samples contained noisy elements due to bioelectric interference and external environment conditions they had to be filtered. The signals contained noise frequencies and motion artefacts induced due to the body posture and motion. The samples had to be extensively filtered using filtering mechanisms to remove wandering and motion artefacts [8].

In order to classify, detect and separate abnormal signals from the normal one's classification models were developed and trained in existing datasets targeting a particular abnormality. In this research, the datasets and software utilities, libraries provided by Physionet Waveform Database (WFDB) were used as shown in Figure 1. The WFDB was used to extract features from ECG and PPG samples. Once the features were obtained machine learning models were developed to train on a given set of features in order to predict abnormality in test samples. A JSON (JavaScript Object Notation) structure that encapsulated the sample readings was adopted which passed the samples to the HAPI (HL7 API) FHIR test server [12] to be logged to EHR if anomalies were detected. The 
ECG and PPG samples encapsulated with JSON data structures were transmitted over 10 seconds' intervals to a HAPI FHIR sandbox test server. HAPI FHIR is Java implementation of FHIR and enables development of RESTful Webservices. The observation object that encapsulates an instance of observation was coded according to SNOMED-CT coding system as shown in Figure 3 [10].

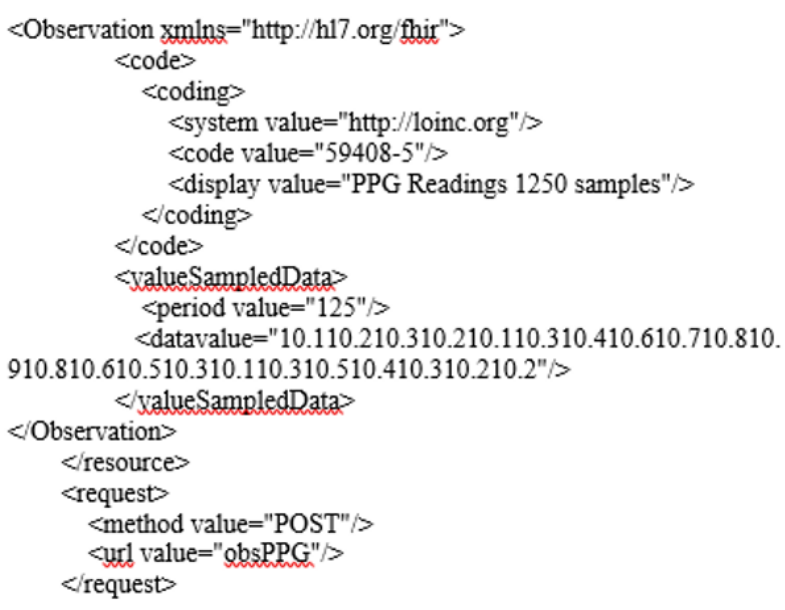

Figure 3: A typical FHIR observation resource showing sampled PPG data with LOINC code 59408-5.

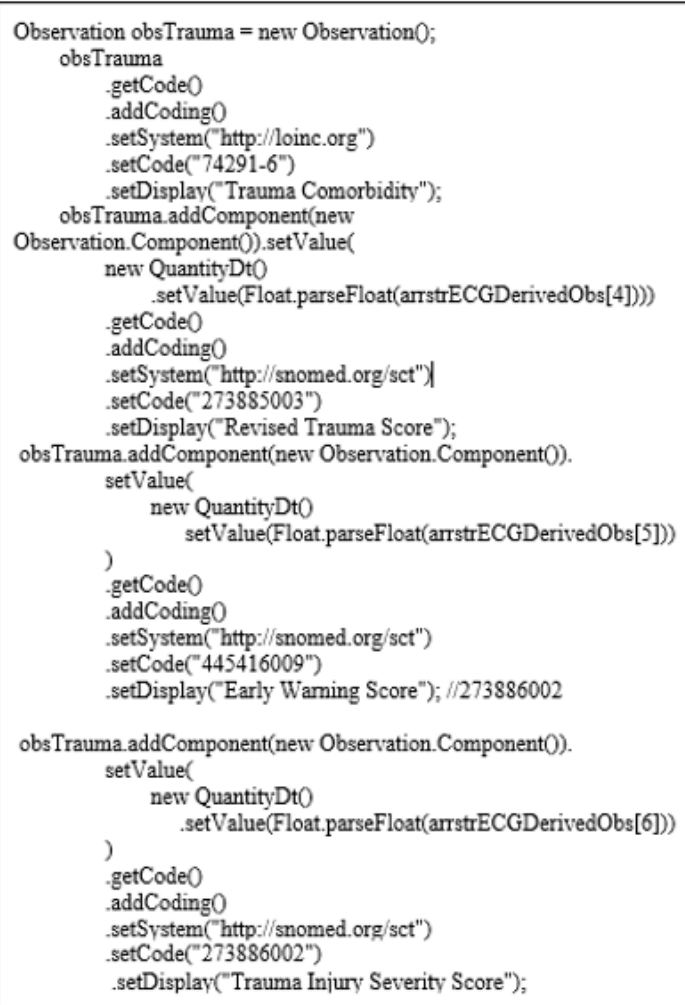

Figure 4: A Trauma event showing modelled as LOINC code 74291-6 with corresponding SNOMED-CT codes for RTS (273885003), EWS (445416009) and TRISS (273886002) scores.
An observation object could be an event of abnormal signal in ECG waveform or a trauma event with trauma scores such as National Early Warning Signs (NEWS), Revised Trauma Scores (RTS), and Trauma Injury Severity Score (TRISS) [9] as shown in Figure 4. The HAPI FHIR data store could be a FHIR based EHR like GP-Connect/GP-SoC from NHS UK [11]. Once abnormal beats or waveforms were detected appropriate alarms could be raised and potentially be passed on to the health-care agency entrusted with the patient care. The FHIR specification on security also supports OAuth authentication and authorization service which the consumer services can embed in their FHIR servers [13]. The clinFHIR tool [14] was used to model observation resources. The composite IoT device modelled as "Device" resource showing reference to observations like ECG, PPG, blood pressure, heart rate has been shown in Figure 5. The trauma scores calculated from the physiological parameters have been shown in Figures 6 \& 7 showing the vital signs being modelled as observations.

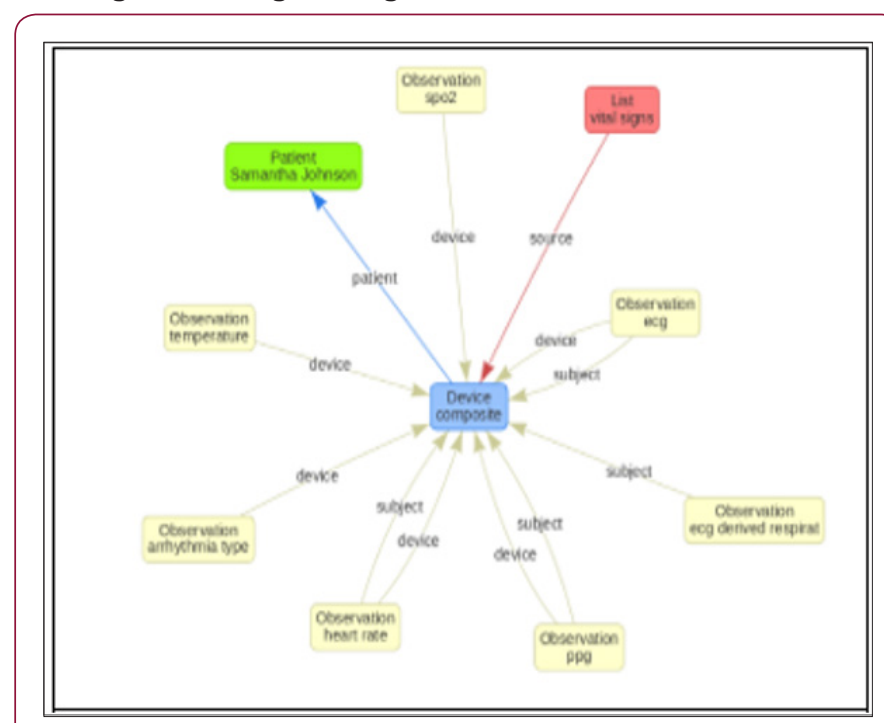

Figure 5: ClinFHIR model for Device that can capture ECG, PPG readings and can calculate vital signs and provide trauma scores when required.

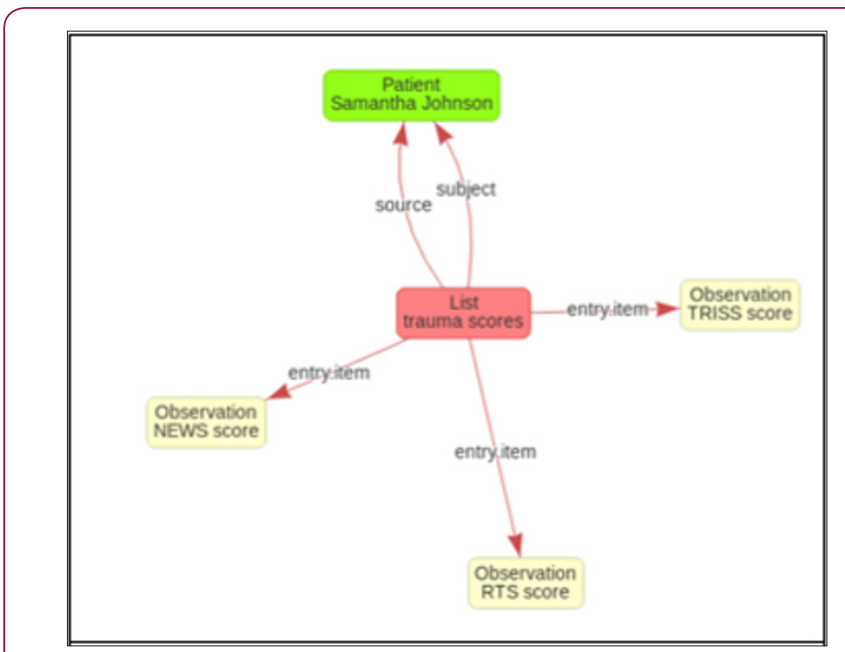

Figure 6: A clinFHIR model for Trauma score observation components. 


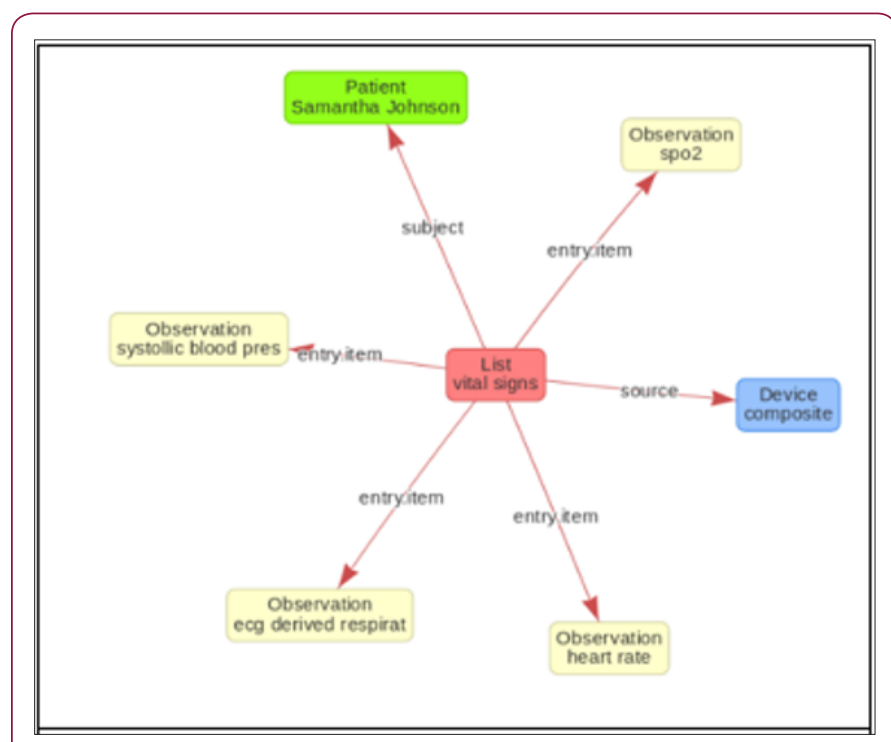

Figure 7: A clinFHIR observation resource for vital signs calculated from physiological parameters.

\section{Conclusions and Discussion}

The motivation behind the research was to provide information on how a real-time data acquisition and data analysis system could be integrated with FHIR client to upload the real time events to FHIR cloud server. An implementation of a HAPI-FHIR test server demonstrated the real-time logging of ECG, PPG, Vital Signs and Trauma observations, according to a Standard Coding System (SNOMED-CT or LOINC), to EHR for further analysis by the general practitioners and medics. This system should assist critical care teams to prepare for an emergency ahead of time and may prevent or reduce hazardous situations. The clinFHIR tool turned out to be an effective tool to model FHIR Device and Observation resources. The tool allowed to build a scenario for real time data acquisition and upload of observations. With further consolidation and standardization of FHIR the monitoring device and observations model could be extended to monitor patients regardless of their location or activity, and would enable uploading trauma related information to FHIR serves hosted by public health services in an automated fashion. The device manufacturers can extend the Observation and Device models to encapsulate FHIR resources.

\section{References}

1. Douglas L Mann, Douglas P Zipes, Peter Libby, Eugene Braunwald, Robert 0 Bonow (2015) Braunwald's Heart Disease: A Textbook of Cardiovascular Medicine, (10 ${ }^{\text {th }}$ Edn.). Elsevier Saunders, USA, p. 1943.

2. Alivecor (2018) Kardiamobile: Peace of mind in your pocket. Leapvault.

3. Shimmer (2018) Individual sensors shimmer.

4. Gackenheimer C (2013) Node.js recipes: A problem-solution approach. Apress, p. 376.

5. Hernández AI, Mora F, Villegas G, Passariello G, Carrault G (2001) Realtime ECG transmission via Internet for nonclinical applications. IEEE Trans Inf Technol Biomed 5(3): 253-257.

6. Mandel JC, Kreda DA, Mandl KD, Kohane IS, Ramoni RB (2016) SMART on FHIR: A standards-based, interoperable apps platform for electronic health records. J Am Med Inform Assoc 23(5): 899-908.

7. Analog Devices (2017) Heart rate monitor for wearable products.

8. Walinjkar A, Woods J (2017) Personalized wearable systems for realtime ECG classification and healthcare interoperability: Real-time ECG classification and FHIR interoperability. Internet Technolo Applic.

9. Walinjkar A (2018) A composite and wearable sensor kit for locationaware healthcare monitoring and real-time trauma scoring for survival prediction. Appl Syst Innov 1(3): 35.

10. Snomed (2017) Code for 3 lead ECG monitoring.

11. NHS Digital (2016) GP System of choice.

12. James A (2018) HAPI FHIR 3.5.0.

13. FHIR (2016) 6.1.0 FHIR Release 3 (STU).

14. Hay D (2017) Hay on FHIR.
ISSN: 2574-1241

DOI: 10.26717/BJSTR.2018.09.001863

Mete Özkıdık. Biomed J Sci \& Tech Res

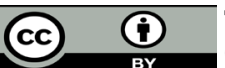

This work is licensed under Creative Commons Attribution 4.0 License

Submission Link: https://biomedres.us/submit-manuscript.php

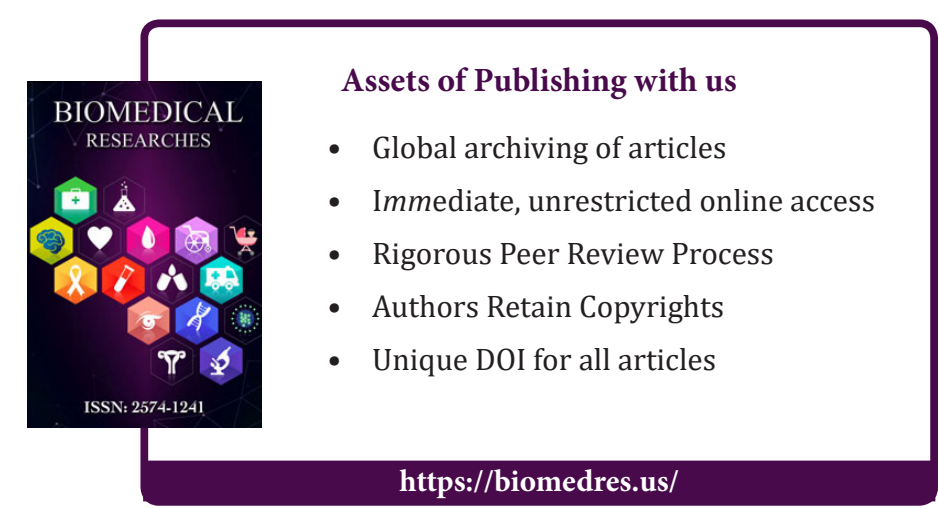

